\title{
Glassy behaviour in short range lattice models without quenched disorder
}

\author{
Mário J. de Oliveira. ${ }^{\dagger}$ and Alberto Petri $\ddagger 1$ \\ † Instituto de Física, Universidade de São Paulo, Caixa Postal 66318, \\ 05315-970 São Paulo SP, Brazil \\ $\ddagger$ Consiglio Nazionale delle Ricerche, Istituto "O.M. Corbino", Via del \\ Fosso del Cavaliere 100, 00133 Roma, Italy \\ and \\ Dipartimento di Fisica and Istituto Nazionale per la Fisica della Materia, \\ Università "La Sapienza", P.le A. Moro 2, 00189 Roma, Italy
}

\begin{abstract}
We investigate the quenching process in lattice systems with short range interaction and several crystalline states as ground states. We consider in particular the following systems on square lattice:

- hard particle (exclusion) model

- $q$ states planar Potts model.

The system is initially in a homogeneous disordered phase and relaxes toward a new equilibrium state as soon as the temperature is rapidly lowered. The time evolution can be described numerically by a stochastic process such as the Metropolis algorithm. The number of pure, equivalent, ground states is $q$ for the Potts model and $r$ for the hard particle model, and it is known that for $r$, or $q \geq d+1$, the final equilibrium state may be polycrystalline, i.e. not made of a uniform phase. We find that in addition $n_{g}$ and $q_{g}$ exist such that for $r>r_{g}$ or $q>q_{g}$ the system evolves toward a glassy state, i.e. a state in which the ratio of the interaction energy among the different crystalline phases to the total energy of the system never vanishes; moreover we find indications that $r_{g}=q_{g}$. We infer that $q=q_{g}$ (and $r=r_{g}$ ) corresponds to the crossing from second order to discontinuous transition in the phase diagram of the system.
\end{abstract}

\footnotetext{
${ }^{1}$ Author to whom correspondence should be addressed; e-mail: petri@idac.rm.cnr.it
} 


\section{Introduction}

A large variety of materials occurs as glass or amorphous (Elliot 1990) but mechanisms driving matter into such kind of states are far from clear. Because of the inherent complexity of the problem, much effort has been devoted also to the investigation of simple models like lattice models. These systems may reproduce at least a few of the characteristic features of glassy behaviour, however they are generally characterized by quenched disorder or competing interactions. In contrast with real glass former such model systems are not able to crystallise and therefore cannot give any information on possible transitions from glassy to crystalline behaviour.

In this work we show that also simple lattice models with short range interaction and no quenched disorder may enter a kind of glassy phase. In particolar we show in section 2 that if a $q$ state Potts system is quenched from infinite to zero temperature, it relaxes toward a state of minimum energy only if $q$ is small enough. Otherwise it slowly equilibrates into a state with energy well above the ground state. Analogous observations on different lattice models are elaborated in section 3 and indicate that such behaviour may be universal.

\section{The Potts model}

Consider a regular lattice in which each site can stay in one of $q$ distinct states, or colours. To each site one may associate a variable $\eta_{i}$ that takes the values $0,1,2, \ldots, q-1$. The energy of a configuration is given by (Potts 1952)

$$
E=-\epsilon \sum_{(i j)}\left(\delta_{\eta_{i}, \eta_{j}}-1\right)
$$

where the summation is over pairs of nearest neighbour sites. If two nearest neighbour sites have the same colour their contribution to the total energy vanishes, otherwise they contribute with an energy $\epsilon$. There are therefore $q$ distinct ground states; in each of them all sites in the lattice have the same colour and the total energy takes the absolute minimum value, $E=0$. These states are crystalline states.

Let $n_{p}$ the number of site pairs such that the two sites have distinct colours, that is

$$
n_{p}=\sum_{(i j)}\left(1-\delta_{\eta_{i}, \eta_{j}}\right)
$$


thus

$$
E=\epsilon n_{p}
$$

and in a crystalline state $n_{p}=0$. A polycrystalline state is composed of clusters of crystalline states of distinct colours separated by $n_{p}$ site pairs of different colours. The number $n_{p}$ however must be small. More precisely we call a state polycrystalline when $\nu=n_{p} / N \rightarrow 0$ as the number of lattice sites $N \rightarrow \infty$, so that the total energy per site $u=E / N \rightarrow 0$, the same of the crystalline state.

We have simulated a quench to zero temperature as follows (Metropolis et al. 1953). At each step of simulation a site is chosen at random and its new colour is chosen at random. If the energy does not increase, the new colour is taken as the new state. The initial configuration of the system is a high temperature one, that is each site has a colour chosen at random.

We have studied the time behaviour of the energy per site $u=E / N$ after the quench on a square lattice for several values of $N=L^{2}$. It is well known that the dynamics of the Potts model usually gets pinned very soon into a non equilibrium state because of the finite size of lattices used in the simulations (Glazier et al. 1990). In order to escape this trap we have used both periodic boundary conditions and fixed boundary conditions. In the latter, all sites on the lattice boundary are frozen in a given state, say the $\eta=0$ state. By this way one can discriminate finite size and boundary effects in the behaviour of the system. In fact we have assumed that, when plotting energy vs time, the superimposing section of curves resulting from different simulation conditions, may be identified with the genuine relaxation of the system. We have extrapolated the behaviour for $L \rightarrow \infty$ from the $1 / \sqrt{(} t)$ part of these curves. As examples, the plots resulting from $q=3$ and $q=7$ are shown in figures 1 and 2 .

From simulations on a square lattice we have drawn the following conclusions:

a) for $q=2$ the system relaxes toward a crystalline state: $n_{p} \rightarrow 0$ and

$$
u \simeq t^{-1 / 2}
$$

b) for $q=3$ the state is polycrystalline: $n_{p} \neq 0$ but $\nu=n_{p} / N \rightarrow 0$,

$$
u \simeq t^{-1 / 2} \text {. }
$$


This agrees with the Lifshitz proposition (Lifshitz 1962) that the final states are non pure crystalline when $q \geq d+1(q \geq 3$ in $d=2)$. However the proposition does not say anything about the energy of these states;

c) for $q>4$ we observe that $u \rightarrow u^{*}>0$ when $t \rightarrow \infty$, since $\nu \rightarrow \nu^{*}>0$, i.e.

$$
u \simeq t^{-1 / 2}+u^{*}
$$

The final state is not polycrystalline and, in addition, its energy is above the energy of the ground state (in fact it is seen to be a monotonic function of $q)$. We call such pinned state a glassy state.

d) for $q=4$ the asymptotic behaviour is well fitted by

$$
u \simeq t^{-1 / 2} \ln (t)
$$

Preliminary results from simulations of quenches at $T>0$ indicate that the observed features persist as far as $T$ is not too large.

\section{Hard core particles on a lattice}

Extension of the above observations to different lattice models, also characterized by short range interaction and absence of quenched disorder, leads to remarkable conclusions. We discuss here some results reported in the literature for a class of lattice gas models, namely exclusion models with random sequential adsorption and in-plane diffusional dynamics.

These models consist of a regular lattice in which particles are placed at the sites of the lattice. The deposition of a particle in a site blocks that site and the sites in a certain neighbourhood, whereas the energy is simply given by

$$
E=-\mu\left(n-n_{c p}\right)
$$

where $\mu>0$ is the chemical potential, $n$ is the total number of deposited particles and $n_{c p}$ is the number of deposited particles in the closest packed configuration. The configuration of closest packing is a ground state configuration because the number of particles gets maximum so that the energy $E$ is minimum $(\mu>0)$ (in analogy with the Potts model the ground state energy is arbitrarily set to zero).

If a particle blocks the deposition of other particles in a certain neighbourhood the ground state is not unique. When for instance nearest neighbours

sites are blocked on a square lattice ( $\mathrm{NN}$ model) then there are two ground 
states corresponding to place the particles on either one or the other of two sub-lattices. If a particle blocks the nearest neighbours as well the next nearest neighbours (NNN model) then there will be four relevant ground states, corresponding to four different sub-lattices. If besides nearest neighbours and next nearest neighbours the particle blocks also the next next nearest neighbours (NNNN model) then the number of ground states will be five.

Once deposited, particles may diffuse along the lattice, with the prescription that they cannot overlap other particles. This process, called diffusional relaxation, makes possible to depose more particles and, in principle, the system enter an ordered phase, coming eventually to the closest packed state where density cannot further increase.

Depending on the model (NN, NNN, NNNN, etc.), the final state may be crystalline, polycrystalline or glassy, and can be classified in a way similar to the case of the Potts model discussed above. Let $n_{s}$ be the number of sites at the surface of clusters of sites in the same state, i.e. occupying the same sub-lattice:

$$
n_{s}=n_{c p}-n \text {. }
$$

In analogy with the Potts model, if $n_{s}=0$ the state is monocrystalline. If $n_{s} \neq 0$ but $n_{s} / N \rightarrow 0$ as $N \rightarrow \infty$, the state is polycrystalline, and is glassy in the opposite case.

We briefly elaborate here on some results reported in the literature by Wang, Nielaba and Privman (1993, 1993a), and by Eisenberg and Baram $(1998,2000)$. In the simulation a lattice initially empty is filled sequentially by placing particles at the non excluded sites. Deposited particles may diffuse to next neighbour sites if these are not blocked or occupied. Although the implementation of this dynamics varies slightly from one author to the other, their results are of general validity. Let us denote by $r$ the number of ground states so that $n_{c p}=N / r$, and let $u=E / N$. By analysing the papers cited above one concludes that:

a) for $\mathrm{r}=2\left(\mathrm{NN}\right.$ model) $n_{s} \rightarrow 0$ and $u \rightarrow 0$ (Wang et al. 1993) since

$$
u_{s} \simeq t^{-1 / 2}
$$

b) for $r=4$ (NNN model), $n_{s} \neq 0$ but $n_{s} / N \rightarrow 0$ as $N \rightarrow \infty$, (Wang et al. 1993a, Eisenberg and Baram 1998) and $u \rightarrow 0$ as

$$
u_{s} \simeq \ln (t) t^{-1 / 2}
$$


c) for $r=5, u \rightarrow u^{*}>0$ and

$$
u_{s} \approx t^{-1}+u_{s}^{*} ; \quad u_{s}^{*}>0 .
$$

This $t^{-1}$ behaviour has been observed in the very late stage (Eisenberg and Baram 2000), when the (finite) system is almost completely filled, and therefore is not to be compared with the asymptotic, size independent, behaviour $t^{-1 / 2}$ of the Potts model. The relevant point here is that $u \rightarrow u^{*}>0$ when $t \rightarrow \infty$, if $r>4$. Frozen states with density lower than in the closest packing have been observed recently also in different exclusion models (Grigera et al. 1997, Fusco et al. 2001).

\section{Discussion and conclusions}

The results obtained for the exclusion model are very similar to those obtained for the Potts model. An important point to stress is that the two models are predicted to have the same symmetries and thus the same critical behaviour (Domany et al. 1977). However they are intrinsically different because there are features of the Potts model, like surface tension and spontaneous magnetization, that are not shared by the hard particle model. Similarities in their behaviour support therefore the idea of $q_{g}=4$ (and $r_{g}=4$ ) as an upper bound for polycrystalline behaviour also in the Potts model.

From the above considerations it can be argued that these different behaviours are actually related to the equilibrium phase diagram of the system. In fact, in two dimensions, for both Potts (Wu 1982) and hard particle models (Runnels 1983) the upper critical number of ground states is four.

Another important point to remark is that the appropriate procedure to get the limiting energy is to take first the thermodynamic limit $L \rightarrow \infty$, and then the infinite time limit $t \rightarrow \infty$. This is a very important point that makes possible the observation of glassy phases in the kind of systems considered here. In fact systems with only short range interaction and no quenched disorder cannot have metastable states in the usual sense (Griffiths 1964, Yeomans 1992).

In conclusion we have shown that dynamics of simple lattice models exhibits a transition from (poly) crystalline to glassy behaviour when the number of ground states increases. Investigations on the Potts model and on exclusion models support the hypothesis that the critical number of states 
at which the transition takes place is related to the equilibrium properties

and, for systems with this symmetry, is four. This work has been supported by grants from CCInt-USP (Brazil) and CNR (Italy).

\section{References}

Domany E., Schick M. and Walker J.S., 1977, Phys. Rev. Lett. 38, 1148.

Eisenberg E. and Baram A., 1998, Europhys. Lett. 44, 168.

Eisenberg E. and Baram A., 2000, J. Phys. A 33, 1729.

Elliot S.R., 1990, Physics of amorphous materials (New York: John Wiley \& Sons Inc.).

Fusco C., Gallo. P., Petri A. and Rovere M., 2001, J. Chem. Phys. 114, 7563 .

Glazier J.A., Anderson M.P. and Grest G.S., 1990, Phil. Mag.

B 62, 615 and refs. therein.

Griffiths R.B., 1964, J. Math. Phys. 5, 1215.

Grigera S.A., Grigera T.S. and Grigera J.R., 1997, Phys. Lett. A, 226. Lifhsitz, I.M., 1962, Zh. Eksp. Teor. Fiz. 42, 1354 (Sov. Phys. JETP 15, 939).

Metropolis N., Rosenbluth A.W., Rosenbluth M.N., Teller, A.H and Teller E., 1953, J. Chem. Phys. 21, 1087.

Potts R.B., 1952, Proc. Camb. Phil. Soc. 48, 106.

Runnels, L.K., 1983 Phase transitions and critical phenomena vol. 8, ed C. Domb and J.L. Lebowitz (New York, Academic) p. 305.

Wang J.S., Nielaba P. and Privman V., 1993, Physica A 199, 527.

Wang J.S., Nielaba P. and Privman V., 1993, Mod. Phys. Lett. B 7, 189.

Wu F.Y., 1982, Rev. Mod. Phys. 54, 235.

Yeomans J.M., 1992, Statistical Mechanics of Phase Transitions (New York: Oxford Un. Press). 


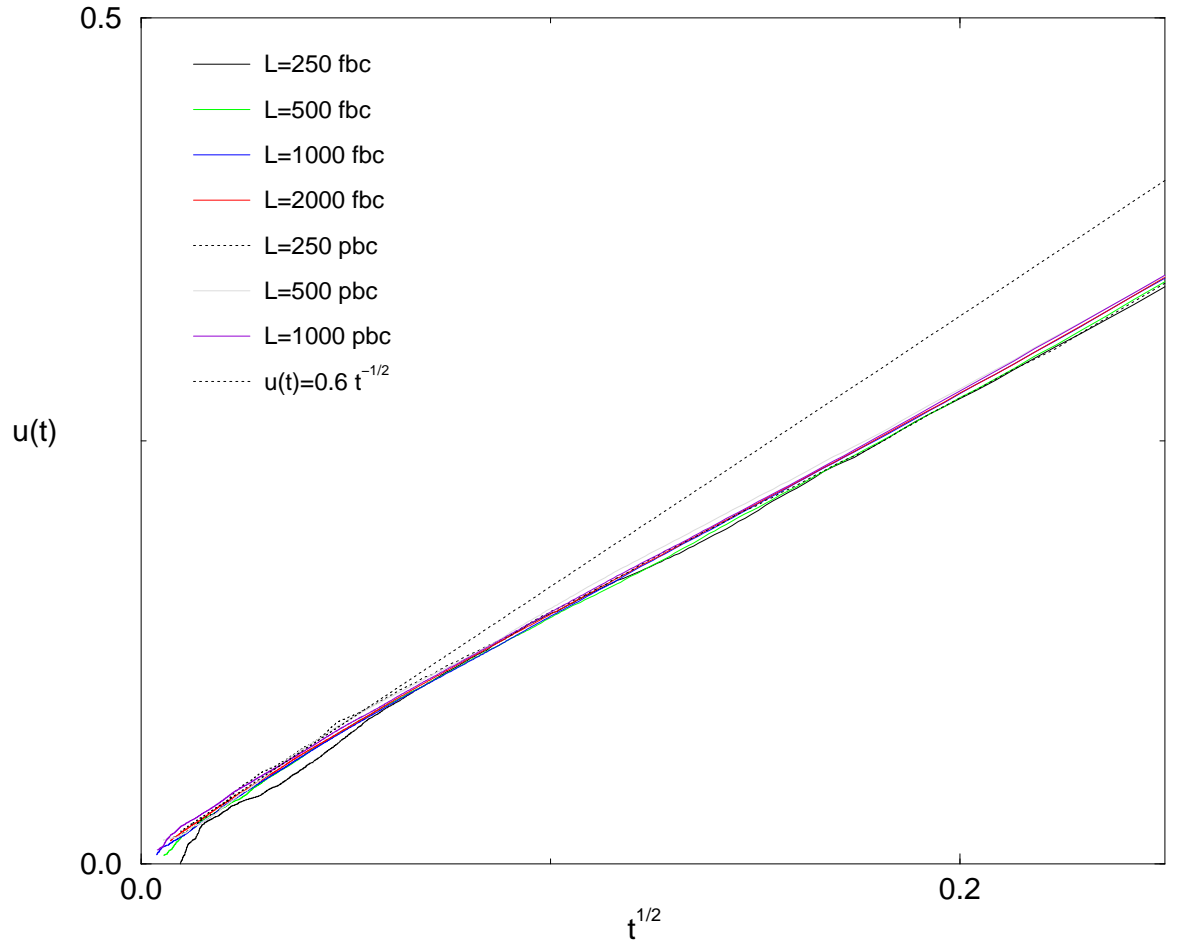

Figure 1: Behaviour of the energy per site with time, for a $q=3$ Potts model on a square lattice. Different curves correspond to different size $(L)$ and boundary conditions $(p b c=$ periodic, $f b c=$ fixed $)$. The dashed line is an extrapolation to $t=\infty$ of the $1 / \sqrt{(} t)$ regime at large times. 


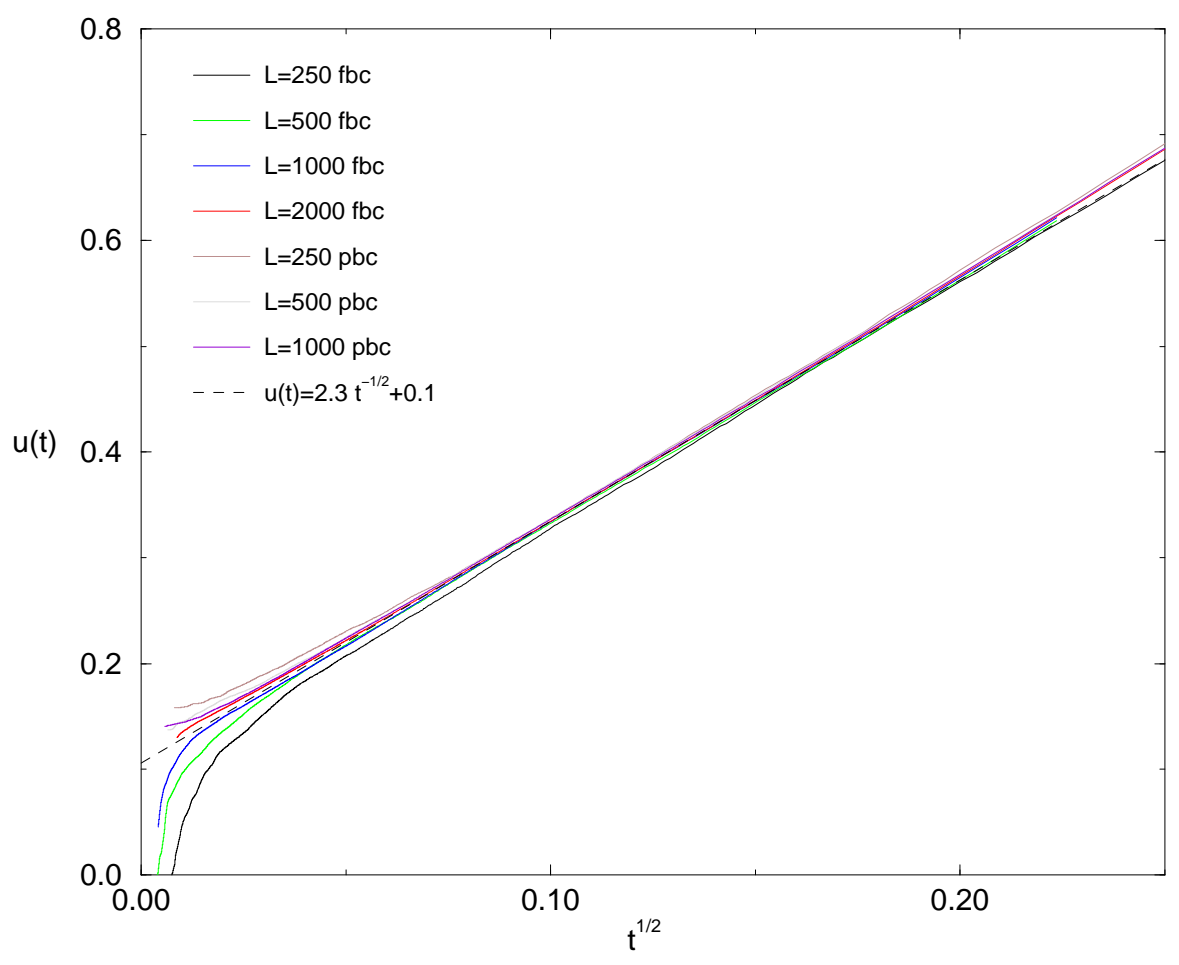

Figure 2: The same as figure 1 for $q=7$. 


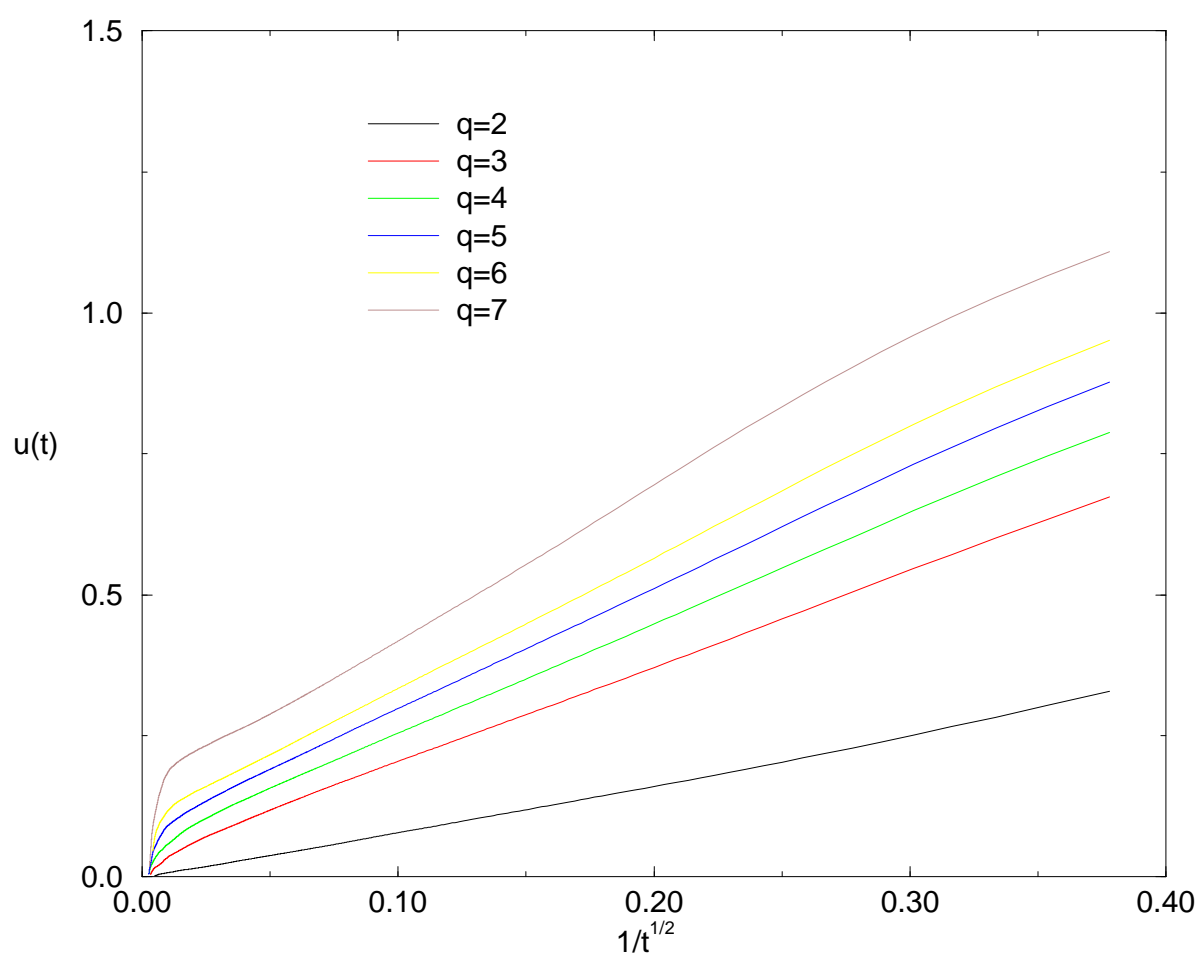

Figure 3: The increase of the excess energy for increasing $q$, when $t \rightarrow \infty$, is well visible from the time behaviour of the energy per site for fixed boundary conditionss $(L=1000)$. 